\title{
Decentralized Computation of Pareto Optimal Pure Nash Equilibria of Boolean Games with Privacy Concerns
}

\author{
Keywords: Boolean Games, Pure Nash Equilibria, Decentralized Learning. \\ Abstract: In Boolean games, agents try to reach a goal formulated as a Boolean formula. These games are attractive \\ because of their compact representations. However, few methods are available to compute the solutions and \\ they are either limited or do not take privacy or communication concerns into account. In this paper we propose \\ the use of an algorithm related to reinforcement learning to address this problem. Our method is decentralized \\ in the sense that agents try to achieve their goals without knowledge of the other agents' goals. We prove that \\ this is a sound method to compute a Pareto optimal pure Nash equilibrium for an interesting class of Boolean \\ games. Experimental results are used to investigate the performance of the algorithm.
}

\section{INTRODUCTION}

The notion of Boolean games or BGs has gained a lot of attention in recent studies (Harrenstein et al., 2001, Bonzon et al., 2006; Bonzon et al., 2007; Dunne et al., 2008; Bonzon et al., 2012; Ågotnes et al., 2013). We explain the concept of a BG with the following example (Bonzon et al., 2006).

\section{Example 1}

Consider the BG with $N=\{1,2,3\}$ the set of agents and $V=\{a, b, c\}$ the set of Boolean action variables. Agent 1 controls $a, 2$ controls $b$ and 3 controls $c$. Each Boolean variable can be set to true or false by the agent controlling it. The goal of agent 1 is $\varphi_{1}=$ $\neg a \vee(a \wedge b \wedge \neg c)$. For agent 2 and 3 we have $\varphi_{2}=a \leftrightarrow$ $(b \leftrightarrow c)$ and $\varphi_{3}=(a \wedge \neg b \wedge \neg c) \vee(\neg a \wedge b \wedge c)$, respectively. We see that each goal is a Boolean proposition and each agent aims to satisfy its own goal, without knowledge of the other agents' goals.

This game could, for instance, be understood as three persons all being able to individually decide to go to a bar (set their variable to true) or to stay home (set their variable to false), without knowing the intentions of others. Given this intuition, the first person either wants to meet the second person without the third or wants to stay home. The second person either wants to meet both the first and third person or wants just one person to go to the bar. The third person's goal is either to only meet the second person or to let the first person be alone in the bar. So generally in BGs, agents try to satisfy an individual goal, which is formulated as a propositional combination of the possible action variables of the agents. A neighbour of an agent $i$ is an agent whose goal depends on an action controlled by agent $i$. In Example 1 all agents are each other's neighbours.

The strength of BGs lies in their compact representation, since BGs do not require utility functions to be explicitly mentioned for every strategy profile. Indeed, utility can be derived from the agents' goals. This advantage also has a downside: computing solutions such as pure Nash equilibria (PNEs) is harder than for most other game representations. Deciding whether a PNE exists in a normal-form game is NPcomplete when the game is represented by the following items: (i) a set of players, (ii) a finite set of actions per player, (iii) a function defining for each player which other players may influence their utility, and (iv) the utility of each player, explicitly given for all joint strageties of the player himself and the players influencing him (Gottlob et al., 2003). Deciding whether a BG has a PNE, on the other hand, is $\Sigma_{2}^{\mathrm{P}}$ complete ${ }^{1}$, even for 2-player zero-sum ${ }^{2}$ games (Bonzon et al., 2006). This high complexity is undoubtedly part of the reason why, to the best of our knowledge, there is no tailor-made method to compute the PNEs of general BGs. However, some techniques are described in the literature related to obtaining solutions of BGs, e.g. finding PNEs for a certain class of BGs or the use of bargaining protocols. These techniques, and the differences from our approach, are discussed in Section 5 Important to note is that, although some of the existing approaches are also decentralized, none of them considers the issues of pri-

\footnotetext{
${ }^{1}$ This is also known as $\mathrm{NP}^{\mathrm{NP}}$-complete, a complexity class at the $2^{\text {nd }}$ level of the Polynomial Hierarchy.

${ }^{2}$ In zero-sum games, the utility of all players sums up to 0 for every outcome.
} 
vacy and limited communication.

In this paper, we approach the problem of computing a PNE of a BG, where we maintain privacy of goals and reduce the amount of communication among the agents. Agents could for instance be unwilling or unable to share their goals, making a centralized approach unsuitable. Moreover, a centralized approach implies higher communication costs, as agents exchange information with a central authority. To cope with these concerns, we investigate a decentralized algorithm, namely Win-Stay Loseprobabilistic-Shift or WSLpS (Mihaylov, 2012). Our solver is private in the sense that agents do not need to communicate their goals, neither to a central authority, nor to each other. To evaluate a goal, it is only required to know what actions were chosen by all relevant agents. Indeed, every agent communicates to every relevant agent its own actions and whether its goal is achieved, without specifying the goal.

The solution we obtain with WSLpS is Pareto optimal, a well-known and desirable property in game theory. An outcome of a game is Pareto optimal if no other outcome makes every player at least as well off and at least one player strictly better off. Depending on the application, an additional advantage is that WSLpS requires only the memory required to represent the current state. An agent does not remember previous actions or outcomes, but only uses the last outcome to choose new actions. As a comparison, e.g. the Highest Cumulative Reward (HCR) (Shoham and Tennenholtz, 1993) update rule in reinforcement learning requires agents to remember the last $l$ chosen actions and outcomes. This advantage of WSLpS could play a crucial role in applications in which agents have a limited memory, e.g. in wireless sensor networks (Mihaylov et al., 2011).

The paper is structured as follows. First, some background on BGs and WSLpS is given in Section 2 In Section 3 we describe how WSLpS can be used to coordinate agents to a Pareto optimal PNE, i.e. to teach agents how to set their action variables in order to satisfy their goal. We prove that this algorithm will converge to a Pareto optimal PNE iff there exists a global strategy for which all agents reach their goal and the parameter choice satisfies an inequality. In Section 4 we present the results of our experiments and in Section 5 we discuss related work. Finally we conclude the paper in Section 6.

\section{PRELIMINAIRIES}

In this section, we recall BGs and WSLpS, the algorithm we will use to find solution of BGs.

\subsection{Boolean Games}

The logical language associated with a set of atomic propositional variables (atoms) $V$ is denoted as $L_{V}$ and contains:

- every propositional variable of $V$,

- the logical constants $\perp$ and $T$, and

- the formulas $\neg \varphi, \varphi \rightarrow \psi, \varphi \leftrightarrow \psi, \varphi \wedge \psi$ and $\varphi \vee \psi$ for every $\varphi, \psi \in L_{V}$.

An interpretation of $V$ is defined as a subset $\xi$ of $V$, with the convention that all atoms in $\xi$ are set to true $(\top)$ and all atoms in $V \backslash \xi$ are set to false $(\perp)$. Such an interpretation can be extended to $L_{V}$ in the usual way. If a formula $\varphi \in L_{V}$ is true in an interpretation $\xi$, we denote this as $\xi \models \varphi$. A formula $\varphi \in L_{V}$ is independent from $p \in V$ if there exists a logically equivalent formula $\psi$ in which $p$ does not occur. The set of dependent variables of $\varphi$, denoted as $D V(\varphi)$, collects all variables on which $\varphi$ depends.

Definition 2.1 (Boolean game (Bonzon et al., 2006)) A Boolean game $(B G)$ is a 4-tuple $G=(N, V, \pi, \Phi)$ with $N=\{1, \ldots, n\}$ a set of agents, $V$ a set of propositional variables, $\pi: N \rightarrow 2^{V}$ a control assignment function such that $\{\pi(1), \ldots, \pi(n)\}$ is a partition of $V$, and $\Phi$ a collection $\left\{\varphi_{1}, \ldots, \varphi_{n}\right\}$ of formulas in $L_{V}$.

The set $V$ contains all action variables controlled by agents. An agent can set the variables under its control to true or false. We adopt the notation $\pi_{i}$ for $\pi(i)$, i.e. the set of variables under agent $i$ 's control (Bonzon et al., 2006). Every variable is controlled by exactly one agent. The formula $\varphi_{i}$ is the goal of agent $i$. For every $p \in V$ we define $\pi^{-1}(p)=i$ iff $p \in \pi_{i}$, so $\pi^{-1}$ maps every variable to the agent controlling it.

Definition 2.2 (Relevant agents, neighbourhood and neighbours)

Let $G=(N, V, \pi, \Phi)$ be a $B G$. The set of relevant variables for agent $i$ is defined as $D V\left(\varphi_{i}\right)$. The set of relevant agents $R A(i)$ for agent $i$ is defined as $\cup_{p \in D V\left(\varphi_{i}\right)} \pi^{-1}(p)$. The neighbourhood of agent $i$ is defined as $\operatorname{Neigh}(i)=\{j \in N: i \in R A(j)\}$. We say that $j$ is a neighbour of $i$ iff $j \in N$ Neigh $(i) \backslash\{i\}$.

\section{Example 2}

Let $G$ be a 2-player BG with $\pi_{i}=\left\{a_{i}\right\}, \varphi_{1}=a_{2}$ and $\varphi_{2}=a_{1} \vee \neg a_{2}$. Then 1 is a relevant agent for 2 , but not for himself. The neighbourhoods in the game are $\operatorname{Neigh}(1)=\{2\}$ and $\operatorname{Neigh}(2)=\{1,2\}$.

Note that the relevant agents for agent $i$ are all agents controlling a variable on which agent $i$ 's goal depends (Bonzon et al., 2007). The neighbours of $i$ are all agents - excluding $i$ - whose goal depends on a variable under agent $i$ 's control. 
Definition 2.3 (Strategy profile)

Let $G=(N, V, \pi, \Phi)$ be a $B G$. For each agent $i \in N$ a strategy $s_{i}$ is an interpretation of $\pi_{i}$. Every n-tuple $S=\left(s_{1}, \ldots, s_{n}\right)$, with each $s_{i}$ a strategy of agent $i$, is a strategy profile of $G$.

Because $\pi$ partitions $V$ and $s_{i} \subseteq \pi_{i}, \forall i \in N$, we also use the set notation $\cup_{i=1}^{n} s_{i} \subseteq V$ for a strategy profile $S=\left(s_{1}, \ldots, s_{n}\right)$. With $s_{-i}$ we denote the projection of the strategy profile $S=\left(s_{1}, \ldots, s_{n}\right)$ on $N \backslash\{i\}$, i.e. $s_{-i}=\left(s_{1}, \ldots, s_{i-1}, s_{i+1}, \ldots, s_{n}\right)$. If $s_{i}^{\prime}$ is a strategy of agent $i$, then $\left(s_{-i}, s_{i}^{\prime}\right)$ is a shorthand for $\left(s_{1}, \ldots, s_{i-1}, s_{i}^{\prime}, s_{i+1}, \ldots, s_{n}\right)$.

The utility function for every agent $i$ follows directly from the satisfaction of its goal.

Definition 2.4 (Utility function)

Let $G=(N, V, \pi, \Phi)$ be a $B G$ and let $S$ be a strategy profile of $G$. For every agent $i \in N$ the utility function $u_{i}$ is defined as $u_{i}(S)=1$ iff $S \models \varphi_{i}$ and $u_{i}(S)=0$ otherwise.

A frequently used solution concept in game theory is the notion of pure Nash equilibrium.

Definition 2.5 (Pure Nash equilibrium)

A strategy profile $S=\left(s_{1}, \ldots, s_{n}\right)$ for a $B G G$ is a pure Nash equilibrium (PNE) iff for every player $i \in N s_{i}$ is a best response to $s_{-i}$, i.e. $u_{i}(S) \geq u_{i}\left(s_{-i}, s_{i}^{\prime}\right), \forall s_{i}^{\prime} \subseteq \pi_{i}$.

The BG in Example 1 has a unique PNE $S=$ $(\emptyset, \emptyset,\{c\})$ or shortly $\{c\}$ in set notation. This means that if the third person goes to the bar, no individual person can change his action to improve the outcome for himself. So the third person cannot improve his own situation by leaving. Similarly the first and second person could decide (individually) to come to the bar, but this individual decision will not lead to a strictly better outcome for them, since they already reach their goal in $S$. In Example 2 the PNEs are $\emptyset,\left\{a_{1}\right\}$ and $\left\{a_{1}, a_{2}\right\}$. In Example 1 and 2, $\{c\}$ resp. $\left\{a_{1}, a_{2}\right\}$ are the unique Pareto optimal PNEs.

\subsection{Win-Stay Lose-probabilistic-Shift}

We now recall Win-Stay Lose-probabilistic-Shift (WSLpS) (Mihaylov, 2012), the algorithm we will use to compute solutions in BGs. WSLpS resembles a reinforcement learning algorithm, because it teaches agents which actions are most beneficial by reinforcing the incentive to undertake a certain action which has been successful in the past. The framework to which it is applied consists of agents, which undertake certain actions in pursuit of a goal. The agents are connected with each other through neighbour relations. Note that this concept of neighbours does not necessarily correspond to Definition 2.2
Using WSLpS, agents try to maximize a function called success. There are multiple ways to choose this function and the idea behind it is that, when every agent has maximized its success function, all agents must have reached their goal. In this paper we assume that the function success, defined for each agent and each strategy profile, only takes the values 0 and 1 . Given a certain strategy profile $S$, each agent evaluates its success function. If its value is 0 (i.e. the agent gets negative feedback), it shifts actions with probability $\beta$. In case of positive feedback, it sticks to its strategy choice. WSLpS was originally introduced to solve normal-form coordination games. In those games, all agents control similar actions and they all try to select a comparable action. For example, if multiple people want to meet each other in a bar, but forget to mention which bar, they should all choose the same bar in order to meet. One could then define an agent $i$ to be successful in iteration it iff every neighbour of agent $i$ has picked a similar action as $i$ in the current strategy profile $S^{i t}$. Another option could be that agent $i$ is successful iff it picked a similar action as a randomly selected neighbour. So in case of the bar meeting example, an agent randomly thinks of a friend and checks whether that friend is in the same bar. If he is, the agent stays in that bar, if not, it goes to a random bar with probability $\beta$.

Initially the agents randomly choose how to act. The strategy profile corresponding with this initial choice is denoted as $S^{0}$, with 0 the iteration number. WSLpS contains a parameter $\alpha \in] 0,1]$, which is used to compute the shift probability $\beta$ in every iteration (see Section 3 ). The probability $\beta$ depends on the agent $i$ and the strategy profile $S^{i t}$ that was chosen in the current iteration it. The stochasitc algorithm WSLpS has converged if, with probability 1 , no agent changes its actions anymore.

\section{APPLYING WSLpS TO BGs}

We can use WSLpS to create an iterative solver for BGs as follows. In the first iteration every agent randomly sets each of the variables under its control to true or false, without knowledge of the actions or goals of the other agents. This can happen simultaneously for all agents. Subsequently every agent $i$ evaluates the outcome. We assume that agents can check whether their own goal is achieved. In some applications it is possible to evaluate a goal without communication between agents, e.g. by observation of the environment. If not, we allow agents to communicate their actions to their neighbours. Further communication between agents is restricted to agents asking their 
neighbours whether their goal is satisfied.

We use an additional parameter $k$, which determines the maximum number of neighbours we take into account to evaluate the success function. With this parameter we can control the amount of communication between the agents. The range of $k$ is $\{1,2, \ldots, n\}$, and if $|N e i g h(i)|<k$, we take $i$ 's entire neighbourhood into account. Therefore, we define $k^{\prime}(i)$ as $\min (k,|N e i g h(i)|)$. We denote the set of all possible subsets of Neigh $(i)$ with $k^{\prime}(i)$ elements as $S S(i, k)$. For every $i \in N$ we define the discretely uniformly distributed random variable $R S(i, k)$, which can take all values in $S S(i, k)$. The binomial coefficient $\left(\begin{array}{c}|N e i g h(i)| \\ k^{\prime}(i)\end{array}\right)$ is the cardinality of $S S(i, k)$. So for any set $r s \in S S(i, k)$, the probability $P(R S(i, k)=r s)$ is $\left(\begin{array}{c}\mid \text { Neigh }(i) \mid \\ k^{\prime}(i)\end{array}\right)$.

For a BG $G=(N, V, \pi, \Phi)$ the function success: $2^{V} \times 2^{N} \rightarrow\{0,1\}$ is defined as $\operatorname{success}(S, r s)=1$ iff $u_{j}(S)=1, \forall j \in r s$ and $\operatorname{success}(S, r s)=0$ iff $\exists j \in r s: u_{j}(S)=0$. For every agent $i \in N$ we define the random variable $\operatorname{success}(S, R S(i, k))$ which takes values in $\{0,1\}$. Specifically, $\operatorname{success}(S, R S(i, k))=1$ with probability $\sum_{r s \in S S(i, k)} P(R S(i, k)=r s \mid \operatorname{success}(S, r s)=1)$. So the random variable $\operatorname{success}(S, R S(i, k))$ is 1 with probability 1 iff for any $k^{\prime}(i)$-sized randomly selected subset $r s$ of Neigh $(i)$ the goal of every agent in $r s$ is satisfied. If we observe the value $r s \in S S(i, k)$ for $R S(i, k)$ in iteration it and success $\left(S^{i t}, r s\right)=1$, this positive feedback leads agent $i$ to keep its current strategy $s_{i}^{i t}$ for the next iteration. If not, the negative feedback drives the agent to independently flip each of the variables under its control with a probability of $\beta(S, i, r s)=\max \left(\alpha-\frac{\left|\left\{j \in r s \mid u_{j}(S)=1\right\}\right|}{k^{\prime}(i)}, 0\right)$. Flipping a variable means setting the corresponding variable to true if it was false and vice versa. Note that $R S(i, k)$ takes a different value in every iteration, but the same value is used by $i$ within one iteration. So to compute the shift probability, the agent uses the same random subset as it did to evaluate its success function.

WSLpS has converged if, with probability 1 , no agent changes its actions anymore. To prove the convergence of WSLpS, we use Markov chains. A finite Markov chain (MC) is a random process that transitions from one state to another, between a finite number of possible states, in which the next state depends only on the current state and not on the sequence of states that preceded it (Grinstead and Snell, 1997). If the transition probabilities between states do not alter over time, the MC is homogeneous. WSLpS, applied to a BG, induces a homogeneous finite MC.

\section{Lemma 3.1}

Let $G=(N, V, \pi, \Phi)$ be a BG. Suppose WSLpS is applied to $G$ and the strategy profile $S$ was chosen in the current iteration. For any strategy profile $S^{\prime}$, the probability of $S^{\prime}$ being chosen in the next iteration only depends on $S$.

Proof. For all strategy profiles $S$ and $S^{\prime}$, define $v\left(S, S^{\prime}, i\right)=\left(\left(S \backslash S^{\prime}\right) \cup\left(S^{\prime} \backslash S\right)\right) \cap \pi_{i} . \quad$ Intuitively $v\left(S, S^{\prime}, i\right)$ is the set of variables under agent $i$ 's control which are either true in $S$ and false in $S^{\prime}$ or the other way around. Let the function $w: 2^{V} \times 2^{V} \times N$ be defined as $w\left(S, S^{\prime}, i\right)=1$ if $v\left(S, S^{\prime}, i\right) \neq \emptyset$ and 0 otherwise. We will abbreviate the notations $R S(i, k)$ and $S S(i, k)$ to resp. $R S$ and $S S$. State $S$ transitions to $S^{\prime}$ iff every agent $i \in N$ changes its strategy $s_{i}$ to $s_{i}^{\prime}$. Therefore it suffices to prove that, for every $i$, the probability of $i$ changing $s_{i}$ to $s_{i}^{\prime}$ only depends on $S$. Take an arbitrary $i \in N$. Then either $v\left(S, S^{\prime}, i\right) \neq \emptyset$, i.e. agent $i$ controls at least one variable that is flipped during a tranistion from $S$ to $S^{\prime}$, or $v\left(S, S^{\prime}, i\right)=\emptyset$, i.e. no variables controlled by $i$ are flipped during a transition from $S$ to $S^{\prime}$. In the first case, $i$ can only change its strategy from $s_{i}$ to $s_{i}^{\prime}$ if we observe a value $r s \in S S$ for $R S$ such that $\operatorname{success}(S, r s)=0$. Moreover, $i$ can only change its strategy to $s_{i}^{\prime}$ if it flips every variable in $v\left(S, S^{\prime}, i\right)$, which happens independently with probability $\beta(S, i, r s)$. For the other variables controlled by $i$, i.e. $\pi_{i} \backslash v\left(S, S^{\prime}, i\right), i$ can only change its strategy to $s_{i}^{\prime}$ if it does not flip any of those variables, which happens independently with probability $1-\beta(S, i, r s)$. Since $R S$ is a discrete random variable, the probability of agent $i$ transitioning from $s_{i}$ to $s_{i}^{\prime}$ is

$$
\begin{aligned}
& \sum_{r s \in S S}(P(R S=r s) \cdot(1-\operatorname{success}(S, r s)) \\
& \left.\cdot \beta(S, i, r s)^{\left|v\left(S, S^{\prime}, i\right)\right|} \cdot(1-\beta(S, i, r s))^{\left|\pi_{i} \backslash v\left(S, S^{\prime}, i\right)\right|}\right) .
\end{aligned}
$$

In case $v\left(S, S^{\prime}, i\right)=\emptyset, i$ can only change its strategy from $s_{i}$ to $s_{i}^{\prime}$ if it does not flips variables. This can happen in two ways: either $\operatorname{success}(S, r s)=0$ but all variables keep their current truth assignment, or $\operatorname{success}(S, r s)=1$. So in this case the probability of agent $i$ transitioning from $s_{i}$ to $s_{i}^{\prime}$ is

$$
\begin{aligned}
& \sum_{r s \in S S}(P(R S=r s) \cdot((1-\operatorname{success}(S, r s)) \\
& \left.\left.\cdot(1-\beta(S, i, r s))^{\left|\pi_{i}\right|}+\operatorname{success}(S, r s)\right)\right) .
\end{aligned}
$$

We can compute the probability of $S$ transitioning to $S^{\prime}$ as

$$
\begin{aligned}
& \prod_{i \in N}\left(( 1 - w ( S , S ^ { \prime } , i ) ) \cdot \sum _ { r s \in S S } \left(P(R S=r s) \cdot \beta(S, i, r s)^{\left|v\left(S, S^{\prime}, i\right)\right|}\right.\right. \\
& \left.\cdot(1-\operatorname{success}(S, r s)) \cdot(1-\beta(S, i, r s))^{\left|\pi_{i} \backslash v\left(S, S^{\prime}, i\right)\right|}\right) \\
& +w\left(S, S^{\prime}, i\right) \cdot \sum_{r s \in S S}(P(R S=r s) \cdot((1-\operatorname{success}(S, r s)) \\
& \left.\left.\left.\cdot(1-\beta(S, i, r s))^{\left|\pi_{i}\right|}+\operatorname{success}(S, r s)\right)\right)\right) .
\end{aligned}
$$


It holds that $P(R S=r s)=|S S(i, k)|^{-1}$ with $|S S(i, k)|=\left(\begin{array}{c}\mid \text { Neigh }(i) \mid \\ k^{\prime}(i)\end{array}\right)$. It is now clear that the transition probability only depends on $S$.

\section{Definition 3.1}

Let $G=(N, V, \pi, \Phi)$ be a $B G$. Then the random process with all strategy profiles of $G$ as possible states and with the probability of state $S$ transitioning to state $S^{\prime}$ given by (1) is called the random process induced by WSLpS applied to $G$ and denoted as $\mathcal{M}_{G}$.

The following property follows immediately from Lemma 3.1 and Definition 3.1

\section{Proposition 3.2}

Let $G=(N, V, \pi, \Phi)$ be a $B G$. Then $\mathcal{M}_{G}$ is a homogenous $M C$ and every iteration of WSLpS applied to $G$ corresponds to a transition of states in $\mathcal{M}_{G}$.

An absorbing state of a MC is a state which transitions in itself with probability 1 . An absorbing MC (AMC) satisfies two conditions: (i) the chain has at least one absorbing state and (ii) for each nonabsorbing state there exists an accessible absorbing state, where a state $u$ is called accessible from a state $v$ if there exists a positive $m \in \mathbb{N}$ such that the probability of state $v$ transitioning in state $u$ in $m$ steps is strictly larger than 0. AMCs have an interesting property (Grinstead and Snell, 1997): regardless of the intitial state, the MC will eventually end up in an absorbing state with probability 1 . As such the theory and terminology of MCs offer an alternative formulation for convergence of WSLpS: WSLpS applied to a BG $G$ converges iff $\mathcal{M}_{G}$ is an AMC.

\section{Proposition 3.3}

Let $G=(N, V, \pi, \Phi)$ be a BG with non-trivial goals, i.e. $R A(i) \neq \emptyset, \forall i \in N$. With $\alpha>\frac{k-1}{k}$, WSLpS applied to $G$ converges iff $G$ has a strategy profile $S$ for which every agent reaches its goal. Moreover, if WSLpS applied to G converges, it ends in a Pareto optimal PNE.

Proof. First note that $S$ is an absorbing state of $\mathcal{M}_{G}$ iff all agents get positive feedback for $S$ with probability 1, i.e. $\operatorname{success}(S, r s)=1, \forall r s \in \cup_{i \in N} S S(i, k)$. We claim this condition is equivalent with $u_{i}(S)=1$ for all $i$. Since every agent must have at least one relevant agent, $\operatorname{success}(S, r s)=1$ for all $r s \in \cup_{i \in N} S S(i, k)$ directly implies $u_{i}(S)=1$ for every $i$. Conversely, if $u_{i}(S)=1$ for all $i$, then all agents reach their goal so in particular every neighbour of every agent reaches its goal, implying success $(S, r s)=1$ for all $r s \in \cup_{i \in N} S S(i, k)$. So $S$ is an absorbing state of $\mathcal{M}_{G}$ iff every agent reaches its goal in $S$. Consequently every absorbing state $S$ of $\mathcal{M}_{G}$ is a Pareto optimal PNE.
WSLpS converges $\Rightarrow \exists S, \forall i \in N: u_{i}(S)=1$

This follows from the previous observation and the fact that convergence of WSLpS applied to $G$ corresponds to $\mathcal{M}_{G}$ ending up in an absorbing state.

WSLpS converges $\Leftarrow \exists S, \forall i \in N: u_{i}(S)=1$ Assume that there exists a strategy profile $S^{g}=\left(s_{1}^{g}, \ldots, s_{n}^{g}\right)$ of $G$ with $u_{i}\left(S^{g}\right)=1$ for every agent $i$. We already reasoned in the beginning of the proof that $S^{g}$ is an absorbing state of $\mathcal{M}_{G}$, so it is sufficient to prove that for each non-absorbing state there exists an accessible absorbing state. In that case $\mathcal{M}_{G}$ is an AMC. Let $S^{1}=\left(s_{1}^{1}, \ldots, s_{n}^{1}\right)$ be an arbitrary non-absorbing state, then $\exists i_{1} \in N$ : $u_{i_{1}}\left(S^{1}\right)=0$. Consequently there exists at least one agent which influences $i_{1}$ (i.e. $R A\left(i_{1}\right) \neq \emptyset$ ) and for every $j \in R A\left(i_{1}\right)$ there exists a $r s \in S S(j, k)$ with $i_{1} \in$ rs. This implies that for every $j \in R A\left(i_{1}\right)$ : $P\left(\operatorname{success}\left(S^{1}, j, R S(j, k)\right)=0 \mid S^{1}, j\right)>0$.

There exists a strategy profile $S^{2}$ such that $s_{j}^{2}=s_{j}^{g}, \forall j \in R A\left(i_{1}\right)$, and for all $j \in N \backslash R A\left(i_{1}\right)$ either $s_{j}^{2}=s_{j}^{1}$ or $s_{j}^{2}=s_{j}^{g}$. Moreover, the probability of $S^{1}$ transitioning to $S^{2}$ is non-zero, due to the following reasons. First, we already reasoned that the probability of all $j \in R A\left(i_{1}\right)$ getting negative feedback from $S^{1}$ is non-zero. Second, all agents with positive feedback from $S^{1}$ stick with their current strategy. Third, all agents with negative feedback can change to any strategy with a non-zero probability; in particular, the probability that all agents $j$, with negative feedback from $S^{1}$, switch to $s_{j}^{g}$ is non-zero. To see this, note that, given we observe the value $r s \in S S(j, k)$ for $R S(j, k)$, the probability of agent $j$ flipping a variable is $\beta\left(S^{1}, j, r s\right)=\max \left(\alpha-\frac{\left|\left\{j^{\prime} \in r s \mid u_{j^{\prime}}\left(S^{1}\right)=1\right\}\right|}{k^{\prime}(j)}, 0\right)$. If agent $j$ got negative feedback from $S^{1}$, then $\operatorname{success}\left(S^{1}, r s\right)=0$ and there must be at least one neighbour $j^{\prime}$ of $j$ with $u_{j^{\prime}}\left(S^{1}\right) \neq 1$. Considering the fact that $\alpha>\frac{k-1}{k}$ and $k^{\prime}(j)=\min (k,|\operatorname{Neigh}(j)|)$, it follows that $\alpha>\frac{k^{\prime}(j)-1}{k^{\prime}(j)}$. Therefore it holds that $\beta\left(S^{1}, j, r s\right)$ is in $] 0,1[$ if agent $j$ got negative feedback from $S^{1}$. So irrespective of whether the transition from $s_{j}^{1}$ to $s_{j}^{2}$ requires flipping variables or not, the transition probability is non-zero. Since $u_{i_{1}}\left(S^{g}\right)=1$ and $s_{j}^{2}=s_{j}^{g}, \forall j \in R A\left(i_{1}\right)$, it follows that $u_{i_{1}}\left(S^{2}\right)=1$. Either $\forall i \in N: u_{i}\left(S^{2}\right)=1$, so $S^{2}$ is an accessible absorbing state, or $\exists i_{2} \in N: u_{i_{2}}\left(S^{2}\right)=0$.

As long as there exists an $i_{l} \in N$ such that $u_{i_{l}}\left(S^{l}\right)=0$ we can find a state $S^{l+1}=\left(s_{1}^{l+1}, \ldots, s_{n}^{l+1}\right)$ such that state $S^{l}$ can transition to $S^{l+1}$ with non-zero probability and such that $s_{j}^{l+1}=s_{j}^{g}, \forall j \in R A\left(i_{l}\right)$, and for all $j \in N \backslash R A\left(i_{l}\right)$ either $s_{j}^{l+1}=s_{j}^{l}$ or $s_{j}^{l+1}=s_{j}^{g}$. 
Moreover, for every $i_{m}$ with $1 \leq m \leq l$ it holds that $u_{i_{m}}\left(S^{l+1}\right)=1$ because $\forall j \in R A\left(i_{m}\right): s_{j}^{l+1}=s_{j}^{g}$. To see this, note that we assumed that every $j \in R A\left(i_{m}\right)$ switched to $s_{j}^{g}$ in the transition from $S^{m}$ to $S^{m+1}$. In all the next transitions, either $j$ kept the previous strategy or switched to $s_{j}^{g}$, so in any case we have $s_{j}^{l+1}=s_{j}^{g}$. Clearly $l=n$ is the maximum value for which $S^{l+1}$ will be an absorbing state accessible from $S^{1}$.

In the basic WSLpS algorithm, agents are altruistic: they take the satisfaction of their neighbours' goals into account to reach a solution. Indeed, if the success function of all relevant agents of $i$ is maximized, then automatically agent $i$ 's goal is satisfied.

We can also consider an alternative function $\operatorname{success}(S, i)=u_{i}(S)$, where agents are self-centered and only check whether their own goal is satisfied. However, using this success function, convergence to a Pareto optimal PNE is no longer guaranteed. Consider e.g the BG in Example 2. There is one global solution $\left\{a_{1}, a_{2}\right\}$, but for initial states $\left\{a_{1}\right\}$ or $\emptyset$ agent 2 has reached its goal and will never alter his action. We can also combine self-centered and altruistic behaviour in the function $\operatorname{success}(S, i, r s)=1$ iff $u_{i}(S)=1$ and $u_{j}(S)=1$ for every $j$ in a random $k^{\prime}(i)$ sized subset $r s$ of neighbours of $i$. It is easy to see that Proposition 3.3 remains valid with this success function.

\section{EXPERIMENTS}

In this section, we investigate the convergence of WSLpS in a number of simulations ${ }^{3}$ All measurements have been performed on a $2.5 \mathrm{GHz}$ Intel Core i5 processor and $4 \mathrm{~GB}$ of RAM. A BG generator was implemented with 3 parameters: (i) the number of agents, (ii) the number of variables controlled by one agent, and (iii) the maximum number of operators appearing in a goal. We randomly generate goals with $\wedge, \vee$ and $\neg$ and make sure there is a solution in which every agent reaches its goal. To this end, we first randomly choose a strategy, and then repeatedly generate clauses. If the clause is satisfied by the strategy it is added as a goal to the problem instance; otherwise we add its negation.

\section{Experiment 1}

In our first experiment we generate one BG $G$ with 30 agents, 10 action variables per agent and a maximum of 15 operators in every goal. We fix the parameter $k$ of WSLpS to 2 and run 1000 tests on $G$ for various

\footnotetext{
${ }^{3}$ The link to implementations and data is hidden due to double blind.
}

values for $\alpha$. Note that Proposition 3.3 only guarantees convergence for $\alpha>0.5$. Figure 1 shows the average number of iterations to convergence in function of $\alpha$, within a $95 \%$ confidence interval of the mean. Note that the $\mathrm{X}$-axis does not have a linear scale.

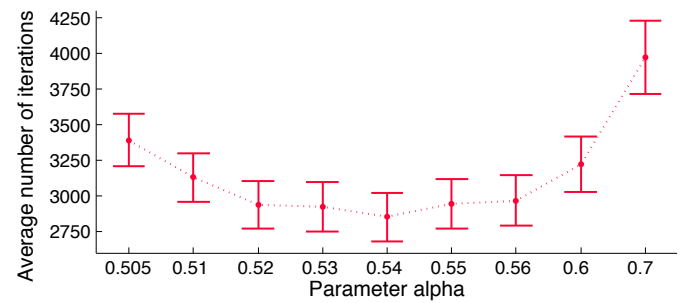

Figure 1: Iterations to convergence in function of $\alpha$.

The best value for $\alpha$ lies close to the boundary for convergence, namely 0.5 . This observation has motivated Experiment 3, where we will investigate more closely how the optimal value for $\alpha$ relates to the theoretical boundary of $\frac{k-1}{k}$.

We see that $\alpha=0.54$ is the best tested value with only 2851 (95\% CI $[2682,3047])$ iterations to convergence on average, with an average computation time of $62 \mathrm{~ms}(95 \%$ CI $[58,66])$. When we used the success function mentioned at the end of Section 3 instead, i.e. $\operatorname{success}(S, i)=1$ iff $u_{i}(S)=1$ and $u_{j}(S)=1$ for every $j$ in a random $k^{\prime}(i)$-sized subset of $i$ 's neighbours, we observed that the convergence was much slower. For $\alpha=0.54$, the average number of iterations to convergence increased substantially to $22 \times 10^{4}(95 \% \mathrm{CI}$ $\left.\left[21 \times 10^{4}, 23 \times 10^{4}\right]\right)$; note that this value is not shown in Figure 1. This increase makes sense: agents start changing their variables when their own goal is not satisfied, even in case they do not influence their own goal. Therefore these changes could drive the agents away from a strategy that contributes to a global solution. So our decentralized approach favors altruistic agents. Hence, if agents cared about others, the whole system will converge much faster, than if agents are self-centered.

\section{Experiment 2}

For this experiment we look at the influence of the ratio of the number of conjunctions to the number of disjunctions per goal. To this end, we use a slightly modified version of our generator, in which we first guess a strategy and then randomly generate clauses with the required number of conjunctions and disjunctions, keeping only those which are satisfied by the strategy. For each tested ratio, we generate one BG with 26 agents and 4 variables per agent. We fix the parameters to $k=2$ and $\alpha=0.54$ and run 1000 tests for all ratios. For the ratio $6 / 0$ we do no reach convergence within $10^{8}$ iterations. Figure 2 shows the average number of iterations to convergence, in a $95 \% \mathrm{CI}$ 
of the mean, scaled logarithmically.

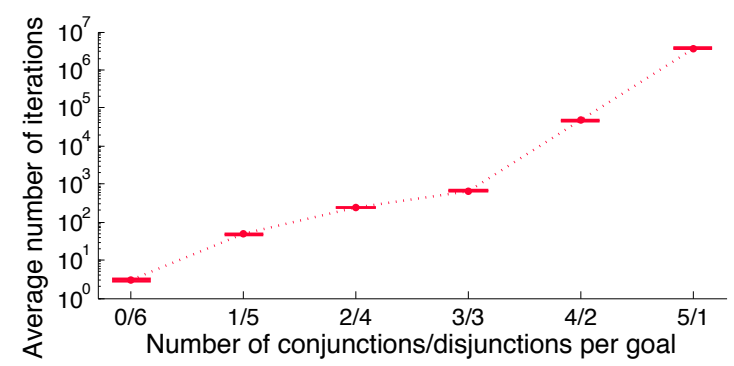

Figure 2: Iterations to convergence for ratios of conjunctions/disjunctions.

If the goals only contain disjunctions, the average number of iterations to convergence is 3 (95\% CI $[2,4])$, with an average computation time of $2.1 \mathrm{~ms}$ $(95 \%$ CI $[2.0,2.2])$. As the number of conjunctions increases compared to the number of disjunctions, the average number of iterations to convergence also increases. These results are intuitive: the more conjunctions in the goals, the fewer absorbing states, so the slower the convergence of WSLpS. Disjunctions have the exact opposite effect. Note, however, that the positions of the operators in the goals and the (number of different) action variables occuring in the goals can also influence the number of absorbing states.

\section{Experiment 3}

In this experiment we investigate the effect of parameter $k$ on the choice of parameter $\alpha$. We have generated one random BG $G$ with 45 agents, 6 action variables per agent and at most 14 operators occuring in every goal. The maximum size of a neighbourhood in $G$ is 12. For each $k$ we determine the best value for $\alpha$ by empirical analysis, up to 2 digits accuracy, the result of which is shown in Figure 3 .

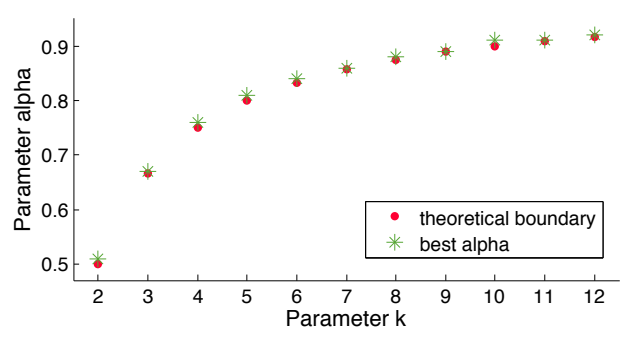

Figure 3: Best $\alpha$ in function of $k$.

We see that the best value for $\alpha$ always lies surprisingly close to the boundary value for convergence we derived in Proposition 3.3. In Figure 4 we plot the average number of iterations to convergence, within a 95\% CI based on 1000 tests, for different values of $k$. For each $k$ we fix $\alpha$ with the values of Figure 3 The average number of iterations to convergence are scaled logarithmically.

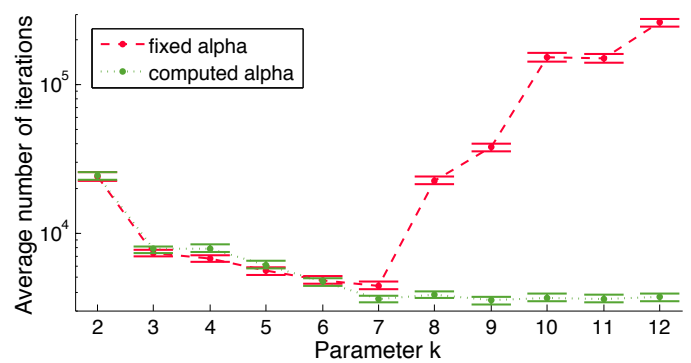

Figure 4: Iterations to convergence in function of $k$.

In Figure 4 we notice something peculiar when the value of $\alpha$ is fixed in this way: as we increase the value of $k$, the average number of iterations to convergence explodes. This is counterintuitive because the higher $k$ is, the more information the agents use to evaluate their success function and choose new actions. However, some agents may have fewer than $k$ neighbours, i.e. $k^{\prime}(i) \neq k$. For those agents another $\alpha$ would be more suitable, but the basic WSLpS algorithm uses the same $\alpha$ for all agents. To analyze this effect, we use an alternative implementation of WSLpS in which every individual agent $i$ computes its own $\alpha$ as floor $\left(\frac{k^{\prime}(i)-1}{k^{\prime}(i)}\right)+0.01$ and we repeat our experiments for different values of $k$. The results are also plotted in Figure 4. This time we get more intuitive results: WSLpS converges faster when more neighbours are asked whether their goal is satisfied. Note however that a higher $k$ implies higher communication costs. One can thus trade off communication costs for convergence speed.

\section{RELATED WORK}

In this section we discuss related work and explain how it differs from ours. One aspect in which it can differ is the studied solution concept. Indeed, there exist many solution concepts of BGs besides PNEs, e.g. the (weak and strong) core (Dunne et al., 2008, Bonzon et al., 2012), verifiable equilibria (Ågotnes et al., 2013) and stable sets (Dunne et al., 2008), although this latter term is also used to describe certain coalitions (Bonzon et al., 2007). In this paper we have restricted the discussion to PNEs, since it is one of the most common, intuitive and straightforward solution concepts in game theory (Daskalakis et al., 2006).

In (Dunne et al., 2008) a bargaining protocol for BGs is introduced. Agents negotiate in rounds by successively proposing an outcome which the others can accept or reject. Under the quite severe restriction that the goals of the agents are positive (i.e. only $\wedge$ and $\vee$ may be used), the negotiations end during the first round and any strategy profile resulting from 
the negotiations is Pareto optimal if the agents follow specific negotiation strategies. If the restriction is not met, the negotiations can last $n$ rounds - with $n$ the number of agents - and obtaining a solution is not guaranteed. This bargaining protocol is decentralized, but has some privacy concerns: it assumes that agents know each other's goals because they try to make a proposal which is at least as good as the previous one for all agents which still need to make a proposal.

In (Bonzon et al., 2007) a centralized algorithm is provided to compute PNEs of BGs for which the irreflexive part of its dependency graph is acyclic. The dependency graph of a BG connects every agent with its relevant agents. In (Wooldridge et al., 2013) taxation schemes are investigated for BGs with cost functions, i.e. BGs extended with costs associated with actions (Dunne et al., 2008). Such a taxation scheme consists of an external agent, which imposes additional costs to incentivise the agents to rationally choose an outcome that satisfies some propositional formula $\Gamma$. For example, one can define a taxation scheme such that the resulting BG has at least one PNE and all PNEs satisfy $\Gamma$. This approach is also centralized: a central entity uses global information to find a taxation scheme.

In (Ågotnes et al., 2013) the privacy of agents is implicitely adressed by extending the BG framework with an individual set of observable actions for every agent. With these sets, a new solution concept of verifiable equilibria is defined. These equilibria differ from others because, when playing the corresponding strategies from the standard notion of Nash equilibrium, agents are actually able to know they have reached an equilibrium. However, the authors assume that the agents can see the complete game: the actions, the goals, who controls which action and who can observe which action. The privacy is thus restricted to the observation of certain actions and the new concept is introduced more from an uncertainty point of view than from a privacy point of view.

\section{CONCLUSION}

We proposed a decentralized approach to find solutions of BGs, based on the WSLpS algorithm. Our method addresses privacy concerns, in the sense that agents are not required to share their goal with each other. We have empirically observed that agents can converge to a global solution with little communication. Moreover, we discovered and analyzed a tradeoff between the convergence speed of WSLpS and the communication costs. We have also proved that, whenever an outcome exists for which every agent reaches its goal and the parameter choice satisfies the restriction $\alpha>\frac{k-1}{k}$, WSLpS converges to a Pareto optimal PNE. Furthermore, simulations have shown that this theoretical boundary for $\alpha$ indicates the most efficient parameter choice, namely by choosing $\alpha$ marginally bigger than $\frac{k-1}{k}$.

\section{REFERENCES}

Ågotnes, T., Harrenstein, P., van der Hoek, W., and Wooldridge, M. (2013). Verifiable equilibria in Boolean games. In Proc. IJCAI'13.

Bonzon, E., Lagasquie-Schiex, M.-C., and Lang, J. (2007). Dependencies between players in Boolean games. In Proc. ECSQARU '07, volume 4724 of LNCS, pages 743-754. Springer.

Bonzon, E., Lagasquie-Schiex, M.-C., and Lang, J. (2012). Effectivity functions and efficient coalitions in Boolean games. Synthese, 187:73-103.

Bonzon, E., Lagasquie-Schiex, M.-C., Lang, J., and Zanuttini, B. (2006). Boolean games revisited. In Proc. ECAI '06, pages 265-269. ACM.

Daskalakis, C., Goldberg, P., and Papadimitriou, C. (2006). The complexity of computing a Nash equilibrium. In Proc. STOC '06, pages 71-78. ACM.

Dunne, P., van der Hoek, W., Kraus, S., and Wooldridge, M. (2008). Cooperative Boolean games. In Proc. AAMAS '08, volume 2, pages 1015-1022. IFAAMAS.

Gottlob, G., Greco, G., and Scarcello, F. (2003). Pure Nash equilibria: hard and easy games. In Proc. TARK '03, pages 215-230. ACM.

Grinstead, C. and Snell, J. (1997). Introduction to Probability. American Mathematical Society.

Harrenstein, P., van der Hoek, W., Meyer, J.-J., and Witteveen, C. (2001). Boolean games. In Proc. TARK '01, pages 287-298. MKP Inc.

Mihaylov, M. (2012). Decentralized Coordination in Multi-Agent Systems. PhD thesis, Vrije Universiteit Brussel, Brussels.

Mihaylov, M., Le Borgne, Y.-A., Tuyls, K., and Nowé, A. (2011). Distributed cooperation in wireless sensor networks. In Proc. AAMAS '11.

Shoham, Y. and Tennenholtz, M. (1993). Co-learning and the evolution of social activity. Technical report, Stanford University.

Wooldridge, M., Endriss, U., Kraus, S., and Lang, J. (2013). Incentive engineering for Boolean games. Artificial Intelligence, 195:418-439. 